\title{
New method for Si-wafer resistivity determination
}

\author{
J. Hájek, V. Papež, M. Horák, \\ Department of Electrotechnology \\ Faculty of Electrical Engineering, CTU in Prague \\ 16627 Prague 6, Czech Republic
}

\begin{abstract}
Wafer resistivity is one of the most important parameters in production of Power Semiconductor Devices (PSD). This parameter is mainly responsible for achieving required breakdown voltage and other electric parameters. Proposal article describes principles and details of newly designed resonant method and new equipment according patent [1] and compares it with commonly known 4point method. This new method is based on resonant measurement of capacity and following calculation. New method exhibits comparable accuracy with 4point method and brings additional advantages.
\end{abstract}

Keywords: resistivity, pn junction, $C$ - $V$ curve, capacity, resonance.

\section{INTRODUCTION}

Silicon wafers are still used in production of PSD. Typical bulk resistivity varies from the order $10 \Omega \mathrm{cm}$ (e.g. welding diodes) up to $300 \Omega \mathrm{cm}$ (e.g. $7 \mathrm{kV}$ rectifier diodes). Typical tolerance of commercially offered wafers is about $10 \%$. Better tolerance and sorting on demand (banding) is charged. Sometimes, suppliers don't want to do banding despite additional tax [2]. The $10 \%$ wide band is too wide and unusable for some applications. Sometimes it is necessary to check the resistivity during or at the end of the production process when it is necessary to reveal the cause of the production defect (e.g. low blocking voltage achieved). For this purpose, a new method is useful that can examine the resistance on pn junction wafers. Such device can be already polarized by external voltage bias.

\section{DESCTRIPTION OF RESONANT METHODE}

\section{Standard method of impedance measurement}

Measurement of voltage dependence of capacity seems to be a trivial issue. Capacity can be measured by common RLC analyzer; voltage bias can be provided by laboratory high-voltage source with output current several hundred of $\mu \mathrm{A}$. In real practice, this approach is not usable. The main limitation is the impossibility to provide reliable separation between RLC analyzer and high voltage circuit. Each fast transient effect in the high voltage part (e.g. spike) is itself transferred to the measuring circuit. In the worst case, the transient is transferred at full voltage level. Voltage breakdown or jump on measured sample will cause overvoltage on the input of RLC analyzer. When measurement is performed with bias voltage in the order of hundreds volts, this overvoltage will certainly damage the used RLC analyzer.

Further, noise generated by measured sample or high voltage source will cause measurement error and increase the uncertainty of measurement. Therefore, new resonant equipment for measuring capacity of biased pn junction with safe separation and reliable measurement has been developed.

\section{Resonant method}

The equipment described in document no. 305210 [1] as industrial design PV 2014-327 serves primary for the measurement of electric capacity of biased pn junction. This capacity is voltage dependent and mostly is decreasing with the square root of applied voltage. Basic principles of equipment are illustrated in the Fig. 1 bellow.

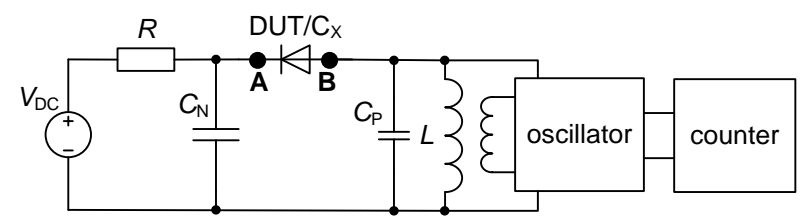

Figure 1: Principles of resonant measurement of capacity.

Measured pn junction (voltage dependent capacity $C_{\mathrm{X}}$ resp. DUT) is connected into resonant circuit which is made by the fixed capacity $C_{\mathrm{N}}$ (vacuum or ceramic capacitor) and by the inductance $L$. Inductance is represented as a parallel combination of ideal coil $L$ and parasitic capacity $C_{\mathrm{P}}$ of winding. Such circuit has its own self resonance frequency given by well-known relation 


$$
f_{r}=\frac{1}{2 \pi \sqrt{L\left(\frac{C_{N} C_{X}}{C_{N}+C_{X}}+C_{P}\right)}}
$$

The self-resonant frequency $f_{\mathrm{r}}$ depends mainly on the capacity of measured junction $C_{\mathrm{X}}$. This capacity $C_{\mathrm{X}}$ can be significantly changed by connecting external voltage bias $V_{\mathrm{DC}}$ through coupling impedance $R$. The applied external DC voltage (bias) does not create any voltage drop across the inductance $L$. Therefore, the polarization voltage on measured sample (DUT) will be exactly the same as on the voltage source $V_{\mathrm{DC}}$. The inductance $\mathrm{L}$ is connected directly to the control circuit of the Meissner oscillator. Oscillator serves for initiating whole circuit into self-resonance. The inductance $L$ has a very low DC resistance and is not loaded by DC polarization voltage. At the same time, it effectively separates the high voltage part of circuit and oscillator by high frequency transformer.

The transformer helps to reduce penetration of energy of avalanche breakdown and other dynamic effects from the high-voltage part of the circuit into evaluating circuit with the oscillator. The resonant circuit also serves as a high efficient band-pass filter and protects the oscillator against overvoltage and other kinds of noise coming from high voltage circuit.

An active device in the oscillator is vacuum tube. This device is more resistant to surges or electronic shocks than semiconductor components. Vacuum tube operates at a higher level of input voltage, so that the effect of noise coming from the measured samples is reduced. The electron-coupled oscillator is made of a pentode, a resonant circuit, a grid resistor and a capacitor. The pentode is equipped by feedback from the second grid. The high-frequency transformer together with the pentode creates a so-called three-point Meissner oscillator. The inductance itself and capacitor in the resonant circuit are designed to be resistant to damage by high-voltage peaks.

The output of the oscillator is indicated on the built-in counter. The actual resonant frequency $f_{\mathrm{r}}$ is than shown immediately for current capacity $C_{\mathrm{X}}$ of the measured sample. From equation (1), then the capacity of measured sample $C_{\mathrm{X}}$ can be simply derived

$$
C_{X}=\frac{1}{\frac{1}{4 \pi^{2} f_{r}^{2} L-C_{P}}-\frac{1}{C_{N}}} .
$$

Knowledge of both main circuit variables $C_{\mathrm{N}}, L$ and parasitic capacity $C_{\mathrm{P}}$ is a necessary condition of the calculation. Parasitic capacity $C_{\mathrm{P}}$ is in the same order as measured $C_{\mathrm{X}}$. Both of them are in the order of hundreds or thousands of picofarads. Therefore, our task is to find out three unknown circuit variables. Fortunately, three independent equations can be written to describe a circuit in three different states as follows:

\section{Disconnected measured sample DUT}

When the terminals A-B are disconnected, the resonant circuit is made only of the inductance $L$ and its own parasitic capacity $C_{\mathrm{P}}$. For such simple circuit we can get the equation 3 :

$$
C_{P}=\frac{1}{4 \pi^{2} f^{2} L} .
$$

Typical resonant frequency of this unloaded circuit is about $1 \mathrm{MHz}$. The parasitic capacity $C_{\mathrm{P}}$ of winding for air-cored coil is of the order of $100 \mathrm{pF}$. Inductors suitable for most of PSDs have an inductance in the order $100 \mu \mathrm{H}$.

\section{Short-circuit measurement of the DUT}

When the terminals A-B are shorted, we basically measure the almost infinite capacity $C_{\mathrm{X}}$. The resonant frequency of such circuit is then given by formula

$$
f_{r}=\frac{1}{2 \pi \sqrt{L\left(C_{N}+C_{P}\right)}} .
$$

The typical resonant frequency of such a circuit will be about $100 \mathrm{kHz}$; using a $C_{\mathrm{N}}$ capacitor of nanofarad units. The $C_{\mathrm{N}}$ capacity should be in the same order (or better higher) then the capacity of measured sample. The capacity $C_{\mathrm{N}}$ cannot be affected by applied polarization voltage; i.e. it must be voltage independent. Therefore, high voltage ceramic disc capacitors designed for output stages of RF transmitters are suitable. Vacuum capacitors usually have a significantly lower capacity, typically in the order of hundreds of picofarads.

\section{General reactance of DUT}

In the general case, the resonant frequency of the circuit is given only by formula (1). Besides semiconductor samples, we can used as the DUT whichever capacity or reactance that will be without losses or losses will be less than $1 \%$. For the determination of main circuit variables, it is useful to use the etalon of capacity (e.g. vacuum capacitor). Such a device has a stable capacity which can be measured by an RLC-bridge with sufficient accuracy.

The described procedure leads to the set of three independent equations with three unknown variables. Such a task can be simply solved with the numerical Solver tools in MS Excel. When more than one etalon with a known capacity is used, we can obtain a larger set of equations that are in mutual dependence. Such a task has a lot of solutions, but with some inaccuracy. There is no analytical solution. The described procedure allows us to verify all parameters of the main circuit before the measurement itself. We can also observe time and temperature shift of these circuit variables. When knowing all parameters of the circuit, we can provide the measurement of $C_{\mathrm{X}}$ according formula (1). 


\section{C-V CURVES, RESISTIVITY CALCULATION}

\section{Description of C-V curves}

When we know the capacity of biased pn junction (mostly at the current DC voltage) we can study the scattering of the electric field in the Space Charge Region (SCR). Finally, we can calculate the initial resistivity of the bulk/Si-wafer. The theoretical background is described in detail e.g. in [3] and can be (with some simplification) shown in the Fig. 2.

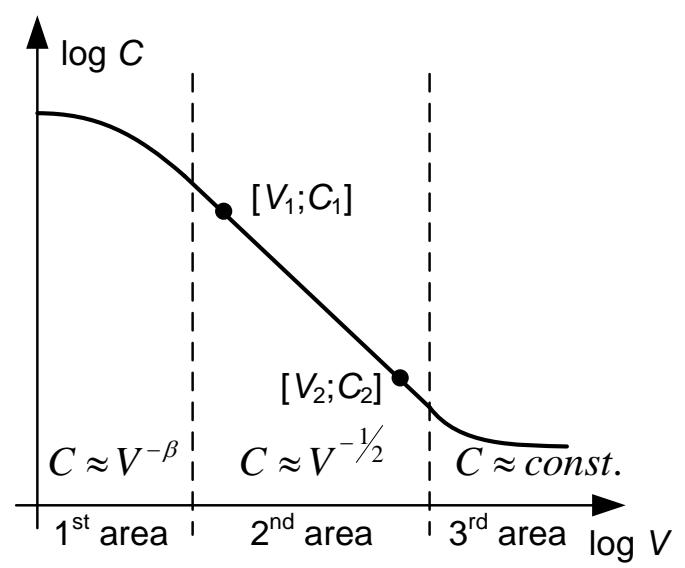

Figure 2: Typical voltage dependence of pn junction capacity in logarithmic scale.

In the $1^{\text {st }}$ area (low polarization voltage in the order units of volts) are capacities of basis $\mathrm{P}$ and $\mathrm{N}$ comparable. The capacity is approximately equal to the power function $C \approx V^{-1 / 3}$. It can be drawn on a logarithmic scale as a decreasing line with the derivation $-1 / 3$. If the applied voltage increases, the capacity behaves as the curve in the $2^{\text {nd }}$ area. The voltage dependence of the capacity is equal to the power function $C \approx V^{-1 / 2}$. Pn junction behaves as an ideal capacitor. The width of SCR, resistivity of initial Si bulk and other parameters can be computed according [3]. On the logarithmic scale, it can be drawn as a declining line with derivation $-1 / 2$. Such a dependence is typical for abrupt pn junction with polarization up to $\mathrm{kV}$ units.

When the applied voltage in the $3^{\text {rd }}$ area continuously increases, the SCR suddenly stopped its expansion. The decrease of capacity slows down. The electric field propagates over the entire width of $\mathrm{Si}$ wafer and has no space for further expansion. The capacity of pn junction stops decreasing and is almost constant.

In the real situation, capacity behaves as a power function $C \approx V^{-\alpha}$, but with a very small exponent approaching zero. This effect is characteristic of $3^{\text {rd }}$ area and is called the „,compressed field“. Such a dependence is typical for power rectifier diodes with narrow bases. They are optimized for rectifying huge currents and have a very low forward voltage drop. Avalanche diodes and high voltage diodes for voltage levels $5 \div 7 \mathrm{kV}$ are characterized only by $2^{\text {nd }}$ area. They behave exactly according theoretical model derived for pn junction.

\section{Resistivity calculation}

In the $2^{\text {nd }}$ area, SCR behaves as an ideal linear plate capacitor with thickness $w$ and relative permittivity $\varepsilon_{\mathrm{r}}=11.7$. Under these conditions, calculations introduced in [3] can be performed. The width of the SCR expansion can be obtained from the formula

$w=\frac{\varepsilon_{0} \varepsilon_{r} S}{C}$,

where $w$ is the width of SCR (typical value about tens of $\mu \mathrm{m}) ; S$ is the active area of pn junction; $C$ is the electrical capacity given by the procedure described in previous chapter. For an abrupt pn junction with $\mathrm{P}^{+} \mathrm{N}$ or $\mathrm{N}^{+} \mathrm{P}$ structure and by Poisson equation, the following expression can be derived

$\frac{1}{C^{2}}=\frac{2}{S^{2} e \varepsilon_{0} \varepsilon_{r} N_{D}}\left(V_{D}+V\right)$,

where $V_{\mathrm{D}}$ is the diffusion voltage; $V$ is the external polarization voltage (bias) and $e$ is the elementary charge. The linear trend of the dependence $1 / C^{2}=f(V)$ follows from the expression (6). Let's note that equations (5) and (6) are correct only for the reverse polarization of the investigated pn junction. Diffusion capacity should be taken into account when forward polarization is assumed. Furthermore, all the expressions dealing with capacity are correct only for a planar pn junction. By combining equations (5) and (6) we can determine the concentration of donors $N_{\mathrm{D}}$ donors in the SCR as follows

$N_{D}=\frac{2 \varepsilon_{0} \varepsilon_{r} V_{r}}{e w^{2}}$

A typical resulting value will be in the range of $10^{12} \mathrm{~cm}^{3}$ to $10^{14} \mathrm{~cm}^{-3}$. The last step of this procedure is the conversion from the $N_{\mathrm{D}}$ concentration to the volume resistivity $\rho(\Omega \mathrm{cm})$. For commonly used material ( $\mathrm{Si}$, $\mathrm{Ge}$, etc.) some empirical formulas or nomograms from old references [4] can be used. There are some online calculators on the websites, e.g. [5]. The simplest empirical formula for converting concentration to volume resistivity follows

$\rho=\frac{4 \cdot 10^{15}}{N_{D}}$.

A circular chip or wafer characterized by its diameter $D$ is the simplest sample of bipolar power device. If we take into account the calculation only in the $2^{\text {nd }}$ area of 
the $C$ - $V$ curve, we can use the expressions (5-8) and assuming a derived dependence $C \approx V^{-1 / 2}$, the resistivity depends only on the dimensions of the sample and its capacity at the given applied voltage.

$\rho=$ const $\cdot \frac{D^{4}}{C^{2} V}=$ const $^{\prime} \cdot D^{4}$

Thus, the calculation of resistivity is affected only by the dimensions of sample (e.g. diameter) and the measured capacity. The result does not depend on the applied voltage; from a physical point of view it cannot depend. Expression (9) is very important for estimating the uncertainty of a method and accuracy.

\section{Electric field compression}

Resonant method resp. the measured dependence $C=f(V)$ can be used for an approximate calculation of compression of the electric filed in the SCR. The possibility to describe all areas of the $C$ - $V$ dependence shown in Fig. 2 by a general power function, where $\beta$ is mostly a negative number, is the basic principle of the method.

The onset of compression is defined as the voltage at which the capacity drop slows. Let's say it is an idealized border between $2^{\text {nd }}$ and $3^{\text {rd }}$ area. This border is actually the intersection of the two curves of the power function. This means that we must solve the following set of equations (10) and (11):

$C=A_{2} \cdot V^{\beta_{2}}$,

$C=A_{3} \cdot V^{\beta_{3}}$.

The coefficients $A_{2}$ and $\beta_{2}$ correspond to the measured dependence in the $2^{\text {nd }}$ area. The coefficients $A_{3}$ and $\beta_{3}$ correspond to the measured dependence in the $3^{\text {rd }}$ area. Using simple algebra, it can be derived that the applied voltage $V$ at the equality of both relations is given by

$V=\left(\frac{A_{2}}{A_{3}}\right)^{\frac{1}{\beta_{3}-\beta_{2}}}$.

The analytical result of (12) has no physical significance. The compression process begins gradually with the expansion of SCR. The border between the $2^{\text {nd }}$ and $3^{\text {rd }}$ areas is not as sharp as shown in the actual measurement in Fig. 3. The compression start value was determined to be $1173 \mathrm{~V}$ according to (12) (marked by gray dashed line).

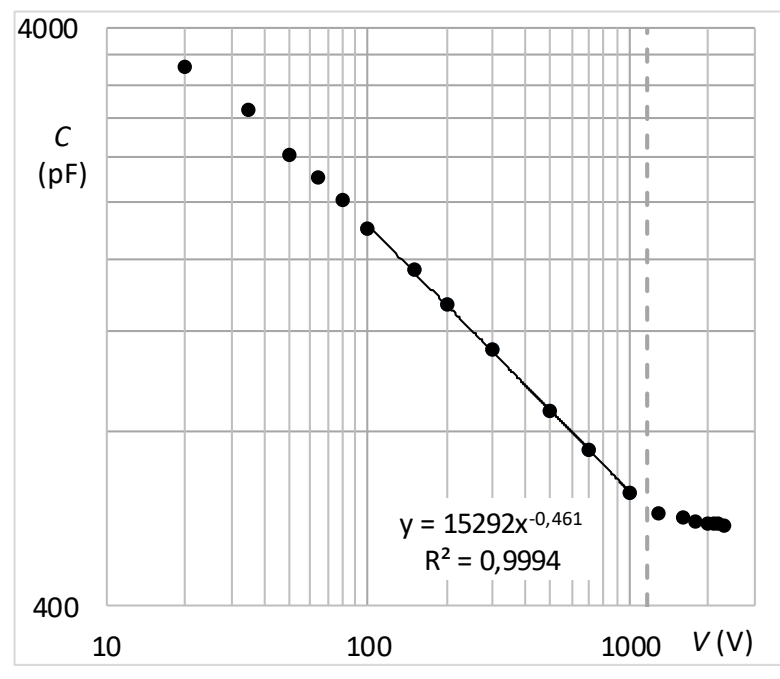

Figure 3: Real measurement of 2,2 kV rectifier diode.

It should be noted that before providing the calculation according to (12), it is necessary to obtain an analytical description of the individual areas of the $C-V$ curve. The process is illustrated in Fig. 2. There are two measured points in the $2^{\text {nd }}$ area marked with coordinates $\left[V_{1} ; C_{1}\right]$ and $\left[V_{2} ; C_{2}\right]$. Substitution of these coordinates into power function $y=A x^{\beta}$ creates a new set of two equations. The analytical solution of this set is trivial:

$\beta=\frac{\log \left(C_{1}\right)-\log \left(C_{2}\right)}{\log \left(V_{1}\right)-\log \left(V_{2}\right)}$

$A=\frac{C_{1}+C_{2}}{V_{1}^{\beta}+V_{2}^{\beta}}$.

The coefficients $A$ and $\beta$ can be than used to calculate the electric field compression according to (12).

\section{PRACTICAL MEASUREMENT AND RESULTS}

A prototype of the equipment described in the previous chapters was built in the year 2018 at the Department of Electrotechnology of the CTU in Prague. The basic parts and layout of the equipment are clearly visible in the Fig. 4. The polarization voltage source is manually controlled in the range $0 \div 7 \mathrm{kV}$ and is equipped with a 4.5-digit display for indication. The operation of the oscillator can be checked by a built-in counter. The oscillator is further connected to the BNC output. The excitation of the vacuum tube circuit can be observed using a built-in micro ammeter. The round table for contacting the measured sample is placed outside the equipment and is fixed with $\mathrm{N}$ connector. This solution shows negligible parasitic capacity and does not affect the measurement. The measuring chamber together with the central table is accessible through a transparent plexiglass cover. 


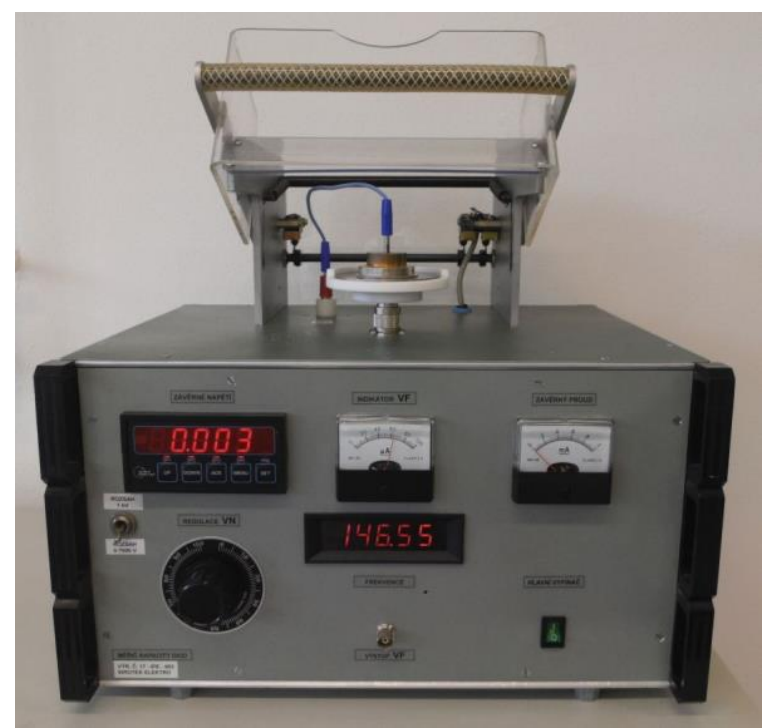

Figure 4: Prototype of a capacity measuring equipment.

The vacuum capacitor shown in the Fig. 5 was used to calibrate and determine all circuit parameters according to the circuit diagram in Fig. 1. A high-voltage vacuum capacitor $100 \mathrm{pF} / 15 \mathrm{kV}$ was made in the Soviet Union for radio broadcasting transmitters. Both capacitor terminals were secured with non-ferromagnetic screw terminals. The capacity of this calibration etalon was measured with an accurate RLC meter HP 4284A with an accuracy of $0.5 \%$.

After that the calibration capacitor was inserted into the measuring chamber and its capacity was measured in the whole range of possible polarization voltage. There are two general advantages to using vacuum capacitor. First, it shows immeasurable power losses over the entire voltage range, i.e. it seems to be an ideal capacitor. Second, the capacity is voltage independent. Such an etalon does not attenuate the resonant circuit and does not affect the measurement itself. The only disadvantage is the relatively small capacity $\left(10^{2} \mathrm{pF}\right)$. Fortunately, this value is comparable to most of the measured samples.

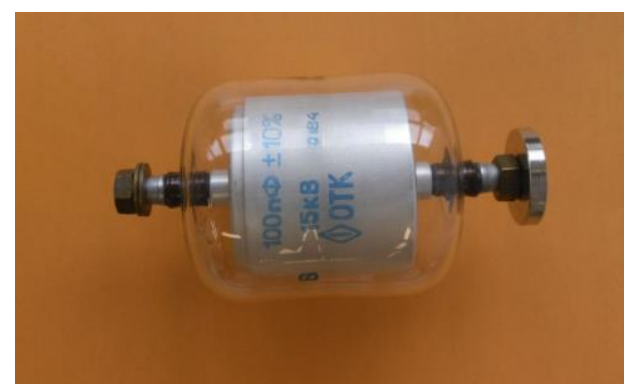

Figure 5: Vacuum capacitor used as an etalon.

Several typical samples of power bipolar devices were measured using the device described in Fig. 4. The first example is introduced at once in Fig. 3. There is a $C-V$ curve typical for rectifier diodes with strong compression of electric filed. A sample $36 \mathrm{~mm}$ in diameter shows a power function in the range of $100 \mathrm{~V}$ to $1000 \mathrm{~V}$ with an exponent close to -0.5 . In the range above $1000 \mathrm{~V}$, a gradual compression of the electric filed occurs. Using formula (12), the limit was calculated as a value of $1170 \mathrm{~V}$. The resistivity of the bulk material was computed based on the measured capacity and its value is $94 \Omega \mathrm{cm}$. The resistivity band required by the $\mathrm{Si}$-wafer supplier is $85 \div 115 \Omega \mathrm{cm}$. In the low-voltage region (tens of volts), the bend of $C-V$ curve corresponding to the $1^{\text {st }}$ area is clearly visible.

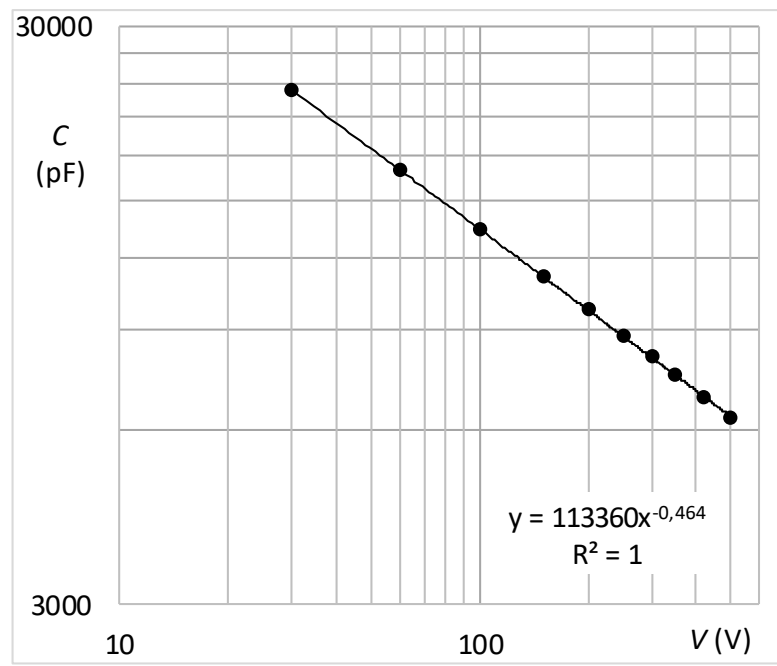

Figure 6: Measured $C$ - $V$ curve of $2.5^{\prime}$ 'welding diode.

Fig. 6 is an example of $C$ - $V$ curve for welding diode with $2.5^{\prime}$ diameter. The sample was measured only in a reduced voltage range up to $500 \mathrm{~V}$. The power dependence has an exponent very close to -0.5 . Large samples $\left(\mathrm{cca} 31 \mathrm{~cm}^{2}\right.$ ) are responsible for a large total capacity of $10^{4} \mathrm{pF}$. The calculated resistivity of the bulk material is $15 \Omega \mathrm{cm}$. This is in a good agreement with the band given by the supplier $10 \div 20 \Omega \mathrm{cm}$. The purpose of welding diodes is to rectify huge current and their reverse blocking voltage usually does not exceed $400 \div 500 \mathrm{~V}$. The $C-V$ curves lie only in the $2^{\text {nd }}$ area and do not show any field compression.

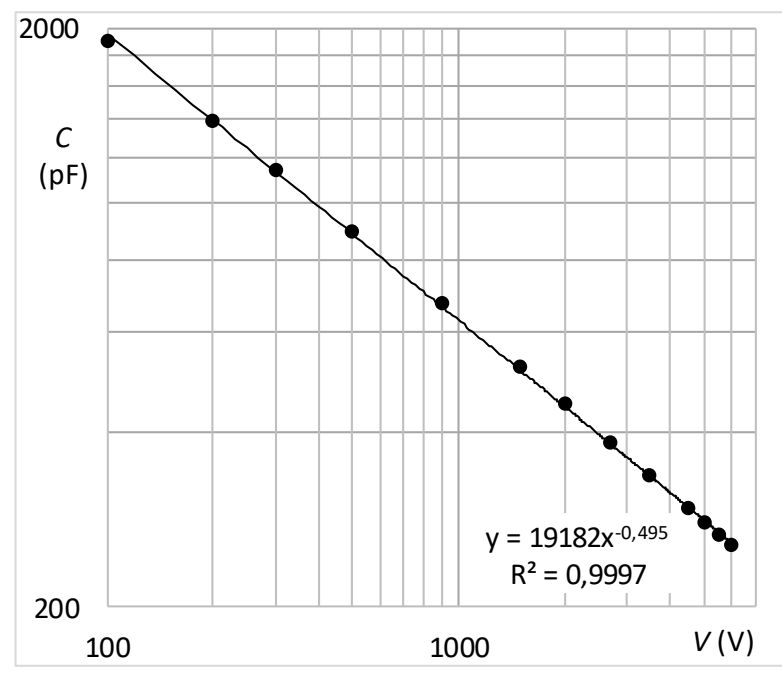

Figure 7: Measured $C$ - $V$ curve of 2' rectifier diode for $6 \mathrm{kV}$. 
Fig. 7 is an example of $C-V$ curve for $6 \mathrm{kV}$ diode with 2 ' diameter. The sample was measured in the whole range up to $7 \mathrm{kV}$. The power function has an exponent exactly equal to -0.5 . The total capacity in the order of $10^{3} \mathrm{pF}$ corresponds to $18 \mathrm{~cm}^{2}$ of active area of the examined sample. The calculated volume resistivity is $270 \Omega \mathrm{cm}$. It is in accordance with the band $245 \div 275 \Omega \mathrm{cm}$ specified by the supplier. Such $6 \mathrm{kV}$ diodes have almost the same profile and $C-V$ character as avalanche diodes. A very thick Si-wafer was used; the initial thickness was more than $800 \mu \mathrm{m}$. Therefore, the whole $C$ - $V$ curve is located only in the $2^{\text {nd }}$ area. There is no field compression.

\section{COMPARISON WITH 4POINT METHOD}

Standard 4point method is based on a direct measurement of the Ohm's resistance of wafer and uses a simplified theoretical model, which is inaccurate in principle. Furthermore, this method needs direct contact with the measured surface, so there is a risk of damages and cracks on the wafers. Simplicity and hardware availability are the biggest advantages. There is a lot of measuring apparatus dedicated directly to this purpose. All manufacturers of Si wafer use this method and their data are comparable. The main disadvantage is that the measurement cannot be performed on the final device. The measurement is only possible on a local volume of cca $\mathrm{mm}^{3}$ before the start of diffusion processes.

The proposed resonant method allows to measure the whole volume of devices and enables measurements on a diffused wafer with a final pn junction. It must be mentioned that the described method cannot be used on a bulk Si-wafer. Triangular electric filed in measured pn junction is needed. Therefore, it can be applied to a diode structure which contains a pn junction allowing an electric field. Statistic control of the diffusion process or reverse engineering on competition devices is possible using this method. This is the main advantage of the newly described method.

The estimation of the inaccuracy of the proposed method has two main parts. The first part deals with the analysis of the resonant circuit and the determination of all parasitic parameters by equations (1-4). The resonant frequency can generally be measured very precisely. Using the described calibration capacitor, we can measure the capacity of the common pn junction (cca $10^{3} \mathrm{pF}$ ) with the accuracy of $\mathrm{pF}$ units. The relative error of capacity measurement is thus $0.5 \%$ or rather $0.1 \%$.

The second part deals with estimating the inaccuracy of the calculation procedure described by the equations (59). An interesting result from (9) is that the largest error concerns the uncertain dimensions of measured sample. The explanation is in Fig. 8. It can be shown that the resistivity is proportional to the $4^{\text {th }}$ power of the diameter $D$ and this parameter has the biggest influence on total inaccuracy of the calculation. The capacity $C_{\mathrm{X}}$ can be measured with an accuracy of $0.5 \%$ or better, see paragraph above.

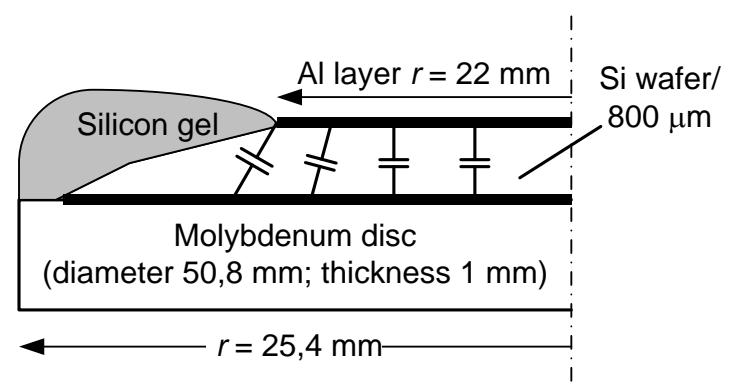

Figure 8: Uncertain diameter of measured capacitor.

The applied polarization voltage $V$ can be measured with an accuracy of $0.1 \%$. The biggest issue is the determination of samples dimensions resp. capacitors dimensions. Remember that only the active area of the sample is important. The chip bevel and facet cover with a silicon coating are not covered by metallization, so they do not participate in the total capacity. According to (9), the total error of the resistivity calculation is in the worst case given by following formula

$\left|\delta_{\rho}\right|=4\left|\delta_{D}\right|+2\left|\delta_{C}\right|+\left|\delta_{v}\right|$,

where $\delta_{\mathrm{1}}$ are relative errors for all variables used. We can conclude that the greatest influence on the uncertainty of resistivity determination has the inaccuracy of determining the sample dimensions.

\section{REFERENCES}

[1] ČVUT v Praze. Fakulta elektrotechnická. Zařizení pro měrení kapacity výkonových vysokonapětových polovodičových součástek. Equipment for high-power, high voltage semiconductor devices capacitance measurement. Původci: V. PAPEŽ. Česká republika. Patent CZ 305210. 2015-04-29.

https://isdv.upv.cz/doc/FullFiles/Patents/FullDocuments/305/3 05210.pdf

[2] http://www.shinetsusilicone-global.com

[3] Frank H., Šnejdar V.: Principy a vlastnosti polovodičových součástek, SNTL, Praha, 1976, 185-186

[4] Nussbaum A.: Fyzika polovodičových součástek, SNTL, Praha, 1965, 319

[5] https://www.pvlighthouse.com.au/resistivity

\section{Addresses of the authors}

Hájek J., CTU in Prague, Technická 2, 16627 Prague, CZ, hajekj1@fel.cvut.cz

Papež V., CTU in Prague, Technická 2, 16627 Prague, CZ, papez@fel.cvut.cz

Horák M., CTU in Prague, Technická 2, 16627 Prague, CZ, horakma4@fel.cvut.cz 